22.

\title{
Propriétés d'un système de courbes algébriques ayant en commun un certain nombre de points.
}

(Par M. Woepcke.)

Nous avons vu (Tome LIII de ce Journal, page 260) que si l'on a un système de trois courbes de l'ordre $n$ passant par $\frac{1}{2} n(n-1)+1+r$ mêmes points, on pourra mener, par les $\frac{1}{2}(n-1)(n+2)-r$ autres intersections de $C_{2}$ avec $C_{3}$ une courbe $c_{1}$ de l'ordre $n-1$ qui coupera, en outre, chacune des deux courbes $C_{2}$ et $C_{3}$ en $\frac{1}{2}(n-1)(n-2)+r$ points, et qu'alors, si par ces deux groupes de $\frac{1}{2}(n-1)(n-2)+r$ points et deux fois $2(n-1)-r$ points, pris respectivement parmi les intersections de $\dot{C}_{1}$ avec $\boldsymbol{C}_{2}$ et $\boldsymbol{C}_{3}$, on mène deux autres courbes $c_{3}$ et $c_{2}$ de l'ordre $n-1$, ces deux courbes passent par $\frac{1}{2}(n-1)(n-2)+r$ mêmes points situés sur la courbe $C_{1}$.

Mais la courbe $c_{3}$ est aussi bien déterminée par les $2(n-1)-r$ intersections de $C_{1}$ avec $C_{2}$ et les $\frac{1}{2}(n-1)(n-2)+r$ points situés sur $C_{1}$ qu'elle a en commun avec $c_{2}$, que par les mèmes $2(n-1)-r$ intersections et les $\frac{1}{2}(n-1)(n-2)+r$, points situés sur $C_{2}$ qu'elle a en commun avec $c_{1}$. On pourra donc aller de la courbe $c_{1}$ à la courbe $c_{2}$, et de celle-ci à la courbe $c_{3}$, qui alors devra passer par les $\frac{1}{2}(n-1)(n-2)+r$ intersections de $c_{1}$ avec $C_{2}$. C'est ce qui nous permet d'énoncer le même théorème de la manière suivante qui se prête facilement aux généralisations que nous allons donner ci-après.

Soient $C_{1}, C_{2}, C_{3}$ trois courbes de l'ordre $n$, passant par $\frac{1}{2} n(n-1)+1+r$ mêmes points, $r$ élant un des nombres $0,1,2, \ldots, 2 n-3$. Par les $\frac{1}{2}(n-1)(n+2)-r$ autres intersections $i_{1,2}$ des courbes $C_{1}$ et $C_{2}$ et par $r$ points pris à volonté sur la courbe $C_{1}$, faisons passer une courbe $c_{1,2}$ de l'ordre $n-1$ qui coupera, en outre, $C_{2}$ en $\frac{1}{2}(n-1)(n-2)+r$ points. Par ceux-ci et $2(n-1)-r$ points pris parmi les $\frac{1}{2}(n-1)(n+2)-r$ points $i_{2,3}$ communs aux courbes $C_{2}$ et $C_{3}$ outre ceux qu'elles ont en commun avec $C_{1}$, menons une courbe $c_{2,3}$ de l'ordre $n-1$; elle passera par toutes les $\frac{1}{2}(n-1)(n+2)-r$ intersections $i_{2,3}$ et coupera, en outre, $C_{3}$ en $\frac{1}{2}(n-1)(n-2)+r$ points. Par ces derniers points et $2(n-1)-r$ points pris parmi les $\frac{1}{2}(n-1)(n+2)-r$ points $i_{1,3}$ communs aux courbes $C_{1}$ et $C_{3}$ 
outre ceux qu'elles ont en commun avec $C_{2}$, menons de nouveau une courbe $c_{1,3}$ de l'ordre $n-1$. Je dis que cette courbe passera par toutes les intersections $i_{1,3}$ et qu'elle passera, en outre, par les $\frac{1}{2}(n-1)(n-2)+r$ intersections de $c_{1,2}$ avec $C_{1}$ différentes des $\frac{1}{2}(n-1)(n+2)-r$ intersections $i_{1,2}$.

Par les $\frac{1}{2} n(n-1)+1+r$ points communs aux courbes $C_{1}, C_{2}, C_{3}$ faisons passer maintenant une quatrième courbe $C_{4}$ de l'ordre $n$. Alors, par les $\frac{1}{2}(n-1)(n-2)+r$ points différents des intersections $i_{2,3}$ en lesquels $C_{3}$ est rencontrée par $c_{2,3}$, nous pourrons mener, outre $c_{1,3}$, une courbe $c_{3,4}$ de l'ordre $n-1$ passant par $2(n-1)-r$ et dès lors par tous les points $i_{3,4}$. De même nous pourrons mener une courbe $c_{1,4}$ par les intersections de $c_{3,4}$ avec $\boldsymbol{C}_{4}$ différentes des intersections $i_{3,4}$ et par $2(n-1)-r$ des points $i_{1,4}$. De ce qui précède il suit que $c_{1,3}$ et $c_{1,4}$ passeront par $\frac{1}{2}(n-1)(n-2)+r$ mêmes points situés sur $C_{1}$ et distincts des intersections $i_{1,3}$ et $i_{1,4}$, et je prétends que ce sont les mêmes $\frac{1}{2}(n-1)(n-2)+r$ points situés sur $C_{1}$ par lesquels passaient déjà $c_{1,3}$ et $c_{1,2}$.

En effet, chacune des courbes $c_{1,2}, c_{1,3}, c_{1,4}$ a en commun avec $C_{1}$ les $\frac{1}{2}(n-1)(n+2)-r$ points $i_{1,2}, i_{1,3}, i_{1,4}$ respectivement, outre lesquels chacune d'elles ne peut avoir en commun avec $C_{1}$ que $\frac{1}{2}(n-1)(n-2)+r$ points. Mais les courbes $c_{1,2}$ et $c_{1,3}$ passent par $\frac{1}{2}(n-1)(n-2)+r$ mêmes points de la courbe $C_{1}$ distincts des points $i_{1,2}$ et $i_{1,3}$, et les courbes $c_{1,3}$ et $c_{1,4}$ passent par $\frac{1}{2}(n-1)(n-2)+r$ mêmes points de la courbe $C_{1}$ différents des points $i_{1,3}$ et $i_{1,4}$. Il faut donc que les $\frac{1}{2}(n-1)(n-2)+r$ points de $C_{1}$ que $c_{1,3}$ a en commun avec $c_{1,2}$ et $c_{1,4}$, soient les mêmes, parceque ces points sont distincts des $\frac{1}{2}(n-1)(n+2)-r$ points $i_{1,3}$ et que la courbe $c_{1,3}$ ne peut rencontrer $C_{1}$ qu'en $n(n-1)$ points.

En faisant un raisonnement analogue pour une cinquième courbe, et enfin pour un nombre quelconque de courbes de l'ordre $n$ qu'on fera passer par les $\frac{1}{2} n(n-1)+1+r$ points communs aux courbes $C_{1}, C_{2}, C_{3}$, on obtient le théorème général suivant.

Si on fait passer $m$ courbes de l'ordre $n$, soient $C_{1}, C_{2}, C_{3}, \ldots, C_{m}$, par $\frac{1}{2} n(n-1)+1+r$ mêmes points, $r$ étant un des nombres $0,1,2, \ldots$, $2 n-3$, il existera, en général, une infinité* $\left.{ }^{*}\right)$ de systèmes de $\frac{1}{2} m(m-1)$ courbes de l'ordre $n-1$, déterminées de la mınière ci-dessus exposée, correspondant respectivement aux autres intersections des courbes $\boldsymbol{C}$ prises

*) Pour $r=0$ il n'existe qu'un seul système de cette espèce. 
deux $\dot{a}$ deux, et dont toujours $m-1$ passent par $\frac{1}{2}(n-1)(n-2)+r$ mềmes points silués sur chacune des courbes $\boldsymbol{C}$.

De mème, si on fait passer m courbes de l'ordre $n$, soient $C_{1}, C_{2}$, $C_{3}, \ldots, C_{m}$, par $\frac{1}{2} n(n+1)+1+r$ mêmes points, $r$ étant un des nombres $0,1,2, \ldots, n-3$, il existera, en général, une infinité de systèmes de $\frac{1}{2} m(m-1)$ courbes de l'ordre $n-2$, correspondant respectivement aux autres intersections des courbes $C$ prises deux à deux, et dont toujours $m-1$ passent par $\frac{1}{2}(n-1)(n-2)+r$ mêmes poinls situés sur chacune des courbes $\boldsymbol{C}$.

Les $\left.\frac{1}{2} m-1\right)$ courbes de l'ordre $n-1$ ou $n-2$ se coupent, trois à trois, en $\frac{1}{6} m(m-1)(m-2)$ groupes de $\frac{1}{2} n(n-1)-r$ ou $\frac{1}{2}(n-2)(n-3)-r$ points, et sur chacune de ces courbes il se trouve $m-2$ de ces groupes.

Il n'est pas besoin de dire que ces deux théorèmes donnent lieu à un très-grand nombre de corollaires et d'applications. Nous n'en citerons ici que quelques - uns des plus simples.

Soient $C_{1}, C_{2}, C_{3}, \ldots, C_{m}$ une série de cercles se coupant en un même point $\boldsymbol{P}$. Sur la circonférence du cercle $\boldsymbol{C}_{\mathbf{1}}$ prenons un point quelconque $p_{1}$; par $p_{1}$ et le second point d'intersection de $C_{1}$ avec $C_{2}$ menons une droite qui coupera $C_{2}$ en un point $p_{2}$; par $p_{2}$ et le second point d'intersection de $C_{2}$ avec $C_{3}$ menons une droite qui coupera $C_{3}$ en $p_{3}$; et ainsi de suite. Je dis que la dernière droite qu'on mène par $\boldsymbol{p}_{m}$ et le second point d'intersection de $\boldsymbol{C}_{m}$ avec $\boldsymbol{C}_{1}$, passera de nouveau par le point $\boldsymbol{p}_{1}$, que le polygone de $m$ côtés ainsi obtenu sera semblable au polygone formé par les centres des cercles, et que chacune de ses diagonales, soit $p_{\alpha} p_{\beta}$, passe par le second point d'intersection de deux des cercles proposés, à savoir $\boldsymbol{C}_{\alpha}$ et $\boldsymbol{C}_{\beta}$.

Soient $\boldsymbol{C}_{1}, \boldsymbol{C}_{2}, \boldsymbol{C}_{3}, \ldots, \boldsymbol{C}_{m}$ une série de coniques ayant un contact du second ordre en un point $\boldsymbol{P}$ et se coupant deux à deux dans un autre point que nous appellerons $i_{\alpha, \beta}$ pour les deux coniques $C_{\alpha}$ et $C_{\beta}$. Sur $C_{1}$ prenons arbitrairement un point $p_{1}$ et menons $p_{1} i_{1,2}$ qui coupera $C_{2}$ en un point $p_{2}$; menons de même $p_{2} i_{2,3}$ qui coupera $C_{3}$ en un point $p_{3}$; et ainsi de suite. La droite $p_{m} i_{1, m}$ viendra repasser par $p_{1}$, et chaque diagonale $p_{\alpha} p_{\beta}$ du polygone $p_{1} p_{2} p_{3} \ldots p_{m}$ passera par un point $i_{\alpha, \beta}$ intersection des coniques $\boldsymbol{C}_{\alpha}$ et $\boldsymbol{C}_{\boldsymbol{\beta}}$.

Soient $C_{1}, C_{2}, C_{3}, \ldots, C_{m}$ une série de courbes du troisième ordre passant par sept mêmes points et se coupant, en outre, deux à deux en deux points. Appelons les deux points d'intersection des courbes $C_{\alpha}$ et $C_{\beta}$ les 
points $i_{\alpha, \beta}$. Les deux points $i_{1,2}$ déterminent une droite $c_{1,2}$, les points $i_{2,3}$ une droite $c_{2,3}$, les points $i_{3,4}$ une droite $c_{3,4}$, et ainsi de suite jusqu'à la droite $c_{m, 1}$. Je dis que ces $m$ droites forment un polygone de $m$ côtés dont chaque sommet se trouve sur une des courbes $\boldsymbol{C}$ respectivement, et que, dans chaque sommet de ce polygone concourent, en outre, $m-3$ autres droites $c$, ou que chaque diagonale du polygone passera par un couple de points $i$.

Prenons un des cas les plus simples de ce dernier corollaire. Soient $C_{1}, C_{2}, C_{3}$ trois cercles passant par deux mêmes points. Sur la circonférence de $C_{1}$ prenons un point quelconque a, de même sur $C_{2}$ un point $b$, et sur $C_{3}$ un point $c$. La droite $a b$ coupera $C_{2}$ en un second point, et la droite ac coupera $C_{3}$ en un second point; , appelons ces deux points $\alpha_{2}$ et $\alpha_{3}$. De même $b a$ coupera $C_{1}$ en un point $\beta_{1}$, et $b c$ coupera $C_{3}$ en un point $\beta_{3}$. Enfin $c a$ coupera $C_{1}$ en un point $\gamma_{1}$ et $c b$ coupera $C_{2}$ en un point $\gamma_{2}$. Je dis que les droites $\alpha_{2} \alpha_{3}$ et $\beta_{1} \beta_{3}$ se rencontreront en un point $\beta_{3}$ situé sur la circonférence de $\boldsymbol{C}_{3}$; que les droites $\alpha_{2} \alpha_{3}$ et $\gamma_{1} \gamma_{2}$ se rencontreront en un point $p_{2}$ situé sur la circonférence de $C_{2}$; et que les droites $\beta_{1} \beta_{3}$ et $\gamma_{1} \gamma_{2}$ se rencontreront en un point $p_{1}$ situé sur la circonférence de $C_{1}$; enfin que le triangle $p_{1} p_{2} p_{3}$ sera semblable au triangle abc.

Faisons encore une application aux courbes du quatrième ordre. Soient $C_{1}, C_{2}, C_{3}$ trois cercles passant par deux mêmes points. Sur la circonférence de $C_{1}$ prenons deux points $a, a^{\prime}$; sur la circonférence de $C_{2}$ deux points $b_{2} b^{\prime}$; et sur la circonférence de $C_{3}$ deux points $c, c^{\prime}$. Le cercle $C_{1}$ et les deux droites $b c, b^{\prime} c^{\prime}$ couperont le cercle $\boldsymbol{C}_{2}$ et les deux droites $a c, a^{\prime} c^{\prime}$ encore en six points $i_{1,2}$; de même $C_{1}, h c, b^{\prime} c^{\prime}$ et $C_{3}, a b, a^{\prime} b^{\prime}$ se couperont encore en six points $i_{1,3}$; et $C_{2}, a c, a^{\prime} c^{\prime}$ et $C_{3}, a b, a^{\prime} b^{\prime}$ se couperont encore en six points $i_{2,3}$. Je dis qu'il existera deux courbes du troisième ordre passant ensemble par six mêmes points situés sur le système $C_{1}, b c, b^{\prime} c^{\prime}$, passant l'une par les six points $i_{1,2}$ et l'autre par les six points $i_{1,3}$, coupant, en outre, l'une le système $C_{2}^{\prime}, a c, a^{\prime} c^{\prime}$ en six points $p_{2}$ et l'autre le système $C_{3}^{\prime}, a b, a^{\prime} b^{\prime}$, en six points $p_{3}$, et se rencontrant enfin en trois points $\pi$ de telle façon que les vingt-un points $i_{2,3}, p_{2}, p_{3}$ et $\pi$ sont situés sur trois droites.

\section{II.}

D'après ce qui a été démontré (Tome LIII, page 260 de ce Journal) trois courbes $C$ de l'ordre $n$ qui passent par $\frac{1}{2} n(n-1)+1+r$ mèmes points, 
donnent lieu à trois courbes $C^{\prime}$ de l'ordre $n-1$ qui, à leur tour, passent par $\frac{1}{2} n(n-1)-r$ mêmes points.

Nous faisons observer que, en vertu de ce mème théorème, si $r<n-1$, les trois courbes $C^{\prime}$ donnent lieu, à leur tour, à trois courbes $C^{\prime \prime}$ de l'ordre $n-2$ qui passent par $\frac{1}{2}(n-2)(n-3)+r$ mêmes points.

De même, si $\boldsymbol{r}>0$, les courbes $C^{\prime \prime}$ donneront lieu à trois courbes $C^{\prime \prime \prime}$ de l'ordre $n-3$ passant par $\frac{1}{2}(n-2)(n-3)-(r-1)$ mêmes points.

Si $r<n-2$, les courbes $C^{\prime \prime \prime}$ donnent pareillement lieu à trois courbes $C^{\text {IV }}$ de l'ordre $n-4$ passant par $\frac{1}{2}(n-4)(n-5)+r-1$ mêmes points, lesquelles, si $r>1$, donnent lieu à trois courbes $C^{\mathrm{v}}$ de l'ordre $n-5$ passant par $\frac{1}{2}(n-4)(n-5)-(r-2)$ mêmes points.

En continuant ainsi on voit qu'on peut arriver à trois courbes de l'ordre $n-2 p$, si l'on fait $r<n-p$ et $>p-2$; et à trois courbes de l'ordre $n-(2 p+1)$, si l'on fait $r<n-p$ et $>p-1$. Conséquemment, pour arriver jusqu'à trois droites, il faut prendre $\frac{n-1}{2}$ ou $\frac{n-3}{2}$ pour $r$, si $n$ est impair, et $\frac{n}{2}$ ou $\frac{n}{2}-1$, si $n$ est pair.

Prenons par exemple trois cercles $C_{1}, C_{2}, C_{3}$ passant par deux mèmes points $\boldsymbol{P}$ et $\boldsymbol{Q}$, et trois droites $\boldsymbol{D}_{1}, \boldsymbol{D}_{2}, \boldsymbol{D}_{3}$ passant par un même point $\boldsymbol{R}$. Le cercle $C_{1}$ et la droite $D_{1}$ couperont, en outre, le cercle $C_{2}$ et la droite $D_{2}$ en quatre points $i_{1,2}$. De même $C_{1}$ et $D_{1}$ couperont $C_{3}$ et $D_{3}$ en quatre points $i_{1,3}$; enfin $C_{2}$ et $D_{2}$ couperont $C_{3}$ et $D_{3}$ en quatre points $i_{2,3}$. Par les quatre points $i_{2,3}$ nous pouvons faire passer (de deux manières différentes) deux droites que nous désignerons ensemble par $c_{2,3}$, et qui couperont le cercle $\boldsymbol{C}_{2}$ en deux points $p_{2}$ et le cercle $C_{3}$ en deux points $p_{3}$. Je dis que les quatre points $i_{1,2}$ et les deux points $p_{2}$ seront sur une conique $c_{1,2}$, que les quatre points $i_{1,3}$ et les deux points $p_{3}$ seront sur ane conique $c_{1,3}$, et que les coniques $c_{1,2}$ et $c_{1,3}$ se couperont en quatre points dont deux seront situés sur les deux droites $c_{2,3}$, et les deux autres sur le cercle $C_{1}$, mais de telle façon que si l'on désigne ces deux derniers points par $p_{1}$, les trois droites quii passent respectivement par les deux points $p_{1}$; les denx points $p_{2}$, et lés deux points $p_{3}$, et que l'on peut désigner respectivement par $c_{1}^{\prime \prime}, c_{2}^{\prime \prime}, c_{3}^{\prime \prime}$, concourent en un même point $\boldsymbol{S}$. 


\section{III.}

Mentionnons encore deux théorèmes relatifs à des lignes droites qui découlent immédiatement du théorème démontré tome LIII, page 260.

1. Soient $\boldsymbol{D}_{1}, \boldsymbol{D}_{2}, \boldsymbol{D}_{3}$ et $\boldsymbol{D}_{1}^{\prime}, \boldsymbol{D}_{2}^{\prime}, \boldsymbol{D}_{3}^{\prime}$ deux faisceaux de droites issus de deux points $\boldsymbol{A}$ et $\boldsymbol{B}$. Appelons $\boldsymbol{i}_{1,2}$ les deux points d'intersection de $\boldsymbol{D}_{1}$ avec $D_{2}^{\prime}$ et de $D_{1}^{\prime}$ avec $D_{2}$; de même appelons $i_{1,3}$ les deux points d'intersection de $D_{1}$ avec $D_{3}^{\prime}$ et de $D_{1}^{\prime}$ avec $D_{3}$; et $i_{2,3}$ les deux points d'intersection de $D_{2}$ avec $D_{3}^{\prime}$ et de $D_{2}^{\prime}$ avec $D_{3}$. Les trois droites $c_{1,2}, c_{1,3}, c_{2,3}$ déterminées respectivement par les couples de points $i_{1,2}, i_{1,3}, . i_{2,3}$ concourront en un même point $\boldsymbol{C}$.

On voit aisément que, de la même manière, le faisceau $C$, ainsi obtenu, donne lieu, à son tour, combiné avec le faisceau $\boldsymbol{A}$, au faisceau $\boldsymbol{B}$; ou combiné avec le faisceau $B$, au faisceau $\boldsymbol{A}$.

De ce théorème on déduit, comme on sait, le théorème corrélatif, que si l'on a sur une droite trois points $a, b, c$ et sur une autre droite trois points $\alpha, \beta, \gamma$, les points d'intersection de $a \beta$ avec $b \alpha$, de $a \gamma$ avec $c \alpha$ et de $b \gamma$ avec $c \beta$ sont en ligne droite.

Si chacun des deux faisceaux se compose de $m$ droites, les points $i$ donnent lieu à $\frac{1}{2} m(m-1)$ droites $c$ qui se coupent, trois à trois, en $\frac{1}{6} m(m-1)(m-2)$ points, et sur chacune des droites $c$ se trouvent $m-2$ de ces points.

2. Par le sommet $a$ d'un triangle $a b c$ menons une droite $\boldsymbol{A}$, par le sommet $b$ une droite $B$, et par le sommet $c$ une droite $C$; appelons $i_{1,2}$ le point d'intersection de $\boldsymbol{A}$ avec $\boldsymbol{B}, i_{1,3}$ le point d'intersection de $\boldsymbol{A}$ avec $\boldsymbol{C}$, et $i_{2,3}$ le point d'intesection de $B$ avec $C$. Par $i_{2,3}$ menons une droite quelconque $c_{2,3}$ coupant $a b$ en $p_{3}$ et $a c$ en $p_{2}$. Je dis que les deux droites $i_{1,2} p_{2}$ et $i_{1,3} p_{3}$ se coupent sur $b c$.

Dessau, au mois de juillet 1857 . 